\title{
ON POST-INSTABILITY PROCESSES IN ADIABATIC SHEAR IN HOT ROLLED STEEL
}

\author{
Da Xing, $\uparrow$ Yi long Bai, Che Min Cheng and Xiao lin Huang \\ Laboratory for Nonlinear Mechanics of Continuous Media, Institute of Mechanics, \\ Chinese Academy of Sciences, Beijing 100080, People's Republic of China
}

(Received 29 August 1990)

\begin{abstract}
EXPERIMENTS carried out using a split Hopkinson torsional bar have shown that only one shear band develops in specimens of hot rolled steel which break during testing. We observed, however, that in specimens which were not deformed to failure, several fine shear bands appeared. We believe that these formed during the loading cycle before the appearance of the final shear band and were not due to the effect of unloading. So we developed a numerical model to study the evolution of shear banding from several finite amplitude disturbances (FADs) in both temperature and strain rate. This numerical model reveals the detailed processes by which the FADs evolve into a fully developed shear band and suggests that beyond instability, the so-called shear banding process consists of two stages : inhomogeneous shearing and true shear-banding. The latter is characterized by the collapse of the stress and an abrupt increase of the local shear strain rate.
\end{abstract}

\section{Notation}

$\tau \quad$ shear stress

$\gamma \quad$ shear strain

$\gamma_{c} \quad$ critical strain for instability to infinitesimal disturbances

$\gamma_{s} \quad$ strain at which disturbances applied (in numerical model)

$\gamma_{l} \quad$ strain for localization

$\dot{\gamma} \quad$ strain rate

$\dot{\gamma}_{0} \quad$ average strain rate

$\theta$ temperature

$\rho$ density

$c$ specific heat

$k$ thermal conductivity

$\beta \quad$ Taylor-Quinney coefficient

$\mu \quad$ stress coefficient in constitutive equation

$m \quad$ strain rate hardening index

$n \quad$ strain hardening index

$v \quad$ thermal softening index

$E$ disturbance energy

$\lambda$ dimensionless wavelength of disturbance

$V_{0}$ boundary velocity

$L \quad$ gauge length

$\dagger$ Present address : PCS, Cavendish Laboratory, Madingley Road, Cambridge CB3 0HE, U.K. 


\section{INTRODUCTION}

NARROW shear bands occur quite frequently in a variety of materials under dynamic loading and are usually termed adiabatic shear bands (RoGERs, 1979). The basic mechanism for them was proposed by ZENER and HoLLOMON (1944) as the thermoplastic instability of material during dynamic loading, caused by thermal softening (due to heat generated by plastic deformation) being stronger than strain hardening, strain rate hardening and other hardening mechanisms. In analytical studies, the maximum shear load criterion (CUlver, 1973; STAKER, 1980) was developed to determine the susceptibility of materials to adiabatic shear banding and a one-dimensional model for simple shear was developed to study the process of dynamic shear. Using this model, instability as well as localization analyses have been carried out (Clifton et al., 1984; Bai, 1982; Fressengeas and Molinari, 1987; Bai et al., 1986; Molinari and Clifton, 1987). Computing codes have also been written to demonstrate various features of adiabatic shear banding (WADA and NAKAMURE, 1978 ; Drew and Flaherty, 1984; Wright and WALTER, 1987 ; BAtra, 1987; KWON and BATRA, 1988).

Although theoretical studies provide some operational rules to predict the onset of shear banding, they do not reveal the whole process. Perhaps the most difficult aspect of adiabatic shear banding is its nonlinearity and transient nature and only small progress on this issue has been made in recent years.

Experimentally, the split Hopkinson torsional bar is one of the most commonly used instruments to investigate the behaviour of materials under dynamic torsional loading (Costin et $a l .$, 1979). Recently, a high-speed infrared radiation detection system and a photographic technique have been used to study the process of shear banding (HARTLey et al., 1987: Marchand) and DufFY, 1988). By using these techniques, MARCHAND and DUfFY (1988) discovered that the process of shear banding consists of three stages: (i) a homogeneous strain state, (ii) an inhomogeneous strain state and (iii) a severe localization stage. In the third stage, severe localized strain quickly leads to a single shear band with the average stress dropping drastically. In a theoretical analysis of the shear banding process, BAI et al. (1986) and WRIGHT and WALTER (1987) pointed out the significance of heat diffusion and plastic work rate in the evolution of the shear band and qualitatively simulated the various stages in adiabatic shear banding.

This paper reports an investigation into the process of shear banding which was carried out both experimentally and by numerical simulation. The experiments performed on a split Hopkinson torsional bar revealed only one fully developed shear band in specimens which broke during testing. Several fine shear bands were observed in specimens which did not break during testing. Based on the assumption that this "multi-fine-shear-band" phenomenon occurred before the appearance of the final shear band and was not an effect caused by unloading, a numerical model was established to study the evolution of shear banding from several finite amplitude disturbances (FADs) in both temperature and strain rate. Our numerical model successfully predicts both the severe increase in the local strain rate and the drastic drop in the stress within the specimen when a shear band fully forms. Moreover, it simulates the complicated processes which occur after the initial instability, namely 
the evolution of several fine shear bands into a single shear band. Our simulation also shows that there exists a nominal strain $\gamma_{l}$ which divides the whole process into two stages, first the coalescence of several fine shear bands and second their localization to form a single shear band. Numerical studies were also carried out to investigate the effects of FADs on the process. These showed that FADs are necessary for the occurrence of severe localization in a finite loading time. The maximum shear load criterion was found to be the lower bound to increasing inhomogeneous shearing.

\section{EXPERIMENT}

\subsection{Description of experimental method}

The tests described were conducted with a split Hopkinson torsional bar. The input and output bars are $25.0 \mathrm{~mm}$ in diameter and made of LY12 aluminium alloy. The connection of the specimen with the input and output bars is different to that used by MARCHAND and DUfFY (1988). In our experiments, we glue the specimen to two connectors and then screw them into the input and output bars. In the tests described here, the rise-time of the loading pulse is about $30-50 \mu$ s and the duration of the pulse is about $1 \mathrm{~ms}$.

Tests were carried out over a range of strains all the way up to specimen failure. The average strain, strain rate and stress in the specimen can be inferred from the strains of the input and output bars by a standard method given by HARTLEY et al. (1985). After testing, the specimens were sectioned and examined with optical microscopes and SEM to study the deformation and microdamage.

Specimens were cut from a 20 \# hot rolled low-carbon steel (20\# HRS) bar, annealed at $900^{\circ} \mathrm{C}$ (see Table 1 for the chemical composition). They were then machined into thin-walled tubes with two circular flanges. The dimensions of the flanges were designed so as to have the same torsional impedance as the aluminium Hopkinson bars.

Figure 1 gives the profile and dimensions of the specimens. Figure 2 gives the texture of the specimen (undeformed and uniformly deformed). The original texture is aligned axially, perpendicular to the shearing direction.

\subsection{Experimental observations}

Three specimens $(3,8$ and 10$)$ were deformed to failure at strain rates in the range $1400-1600 \mathrm{~s}^{-1}$ and nominal strains of about 1.25-1.40. Specimens 5 and 9 deformed "uniformly" (from the macroscopic point of view) at strain rates of $1270-1450 \mathrm{~s}^{-1}$,

TABLE 1. Chemical composition of $20 \#$ hot rolled lowcarbon steel (wt\%)

\begin{tabular}{ccccc}
\hline $\mathrm{C}$ & $\mathrm{Mn}$ & $\mathrm{P}$ & $\mathrm{S}$ & $\mathrm{Si}$ \\
\hline 0.210 & 0.520 & 0.016 & 0.011 & 0.340 \\
\hline
\end{tabular}




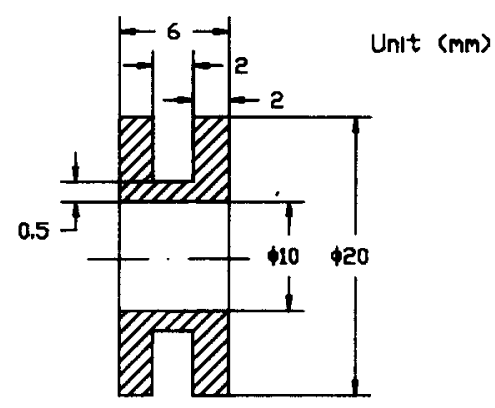

FIG. 1. Dimensions and profile of specimen.

up to nominal strains of about 0.94-1.13. Specimen 11 was deformed at a strain rate of about $700 \mathrm{~s}^{-1}$, to a nominal strain of 0.55 . The stress-strain curves and strain ratenominal strain curves of these six tests are shown in Fig. 3a and b. Table 2 summarizes these six tests.

Although at first glance, the scribe lines showed uniform shear deformation across the gauge section in specimens 5 and 9, metallography revealed several fine shear bands or nonuniformities parallel to the direction of shear within the deformed region (Fig. 4a and b). Very interestingly, in specimens 3, 8 and 10 (which were deformed to failure), only one fully developed shear band (about $200-400 \mu \mathrm{m}$ wide) appears at a nominal strain of about 1.40 (see Fig. 5), when the stress drops severely and fracture occurs.

\subsection{Some results of our experiment}

For 20 \# HRS specimens loaded on the split Hopkinson torsional bar at a strain rate of about $1270-1600 \mathrm{~s}^{-1}$, the following three features are worth emphasizing:

1. The nominal strain at the maximum of the stress-strain curve is in the range $0.65-0.88$ (Table 2). This corresponds to the critical strain given by the maximum shear loading criterion and the criterion from infinitesimal perturbation analysis.

TABLE 2

\begin{tabular}{ccccc}
\hline Specimen & $\begin{array}{c}\text { Deformation } \\
\text { mode }\end{array}$ & $\dot{\gamma}^{\left(\mathrm{s}^{-1}\right)}$ & $\gamma\left(\right.$ at $\left.\tau_{\max }\right)$ & $\begin{array}{c}\text { Nominal } \\
\text { strain }\end{array}$ \\
\hline 03 & Failed & 1600 & $0.76-0.80$ & 1.40 \\
08 & Failed & 1550 & $0.75-0.80$ & 1.25 \\
10 & Failed & 1400 & $0.65-0.75$ & 1.25 \\
05 & MFSB $\dagger$ & 1450 & $0.78-0.88$ & 1.26 \\
09 & MFSB & 1270 & $0.65-0.74$ & 1.12 \\
$11^{+}$ & Uniform & 700 & & 0.62 \\
\hline
\end{tabular}

+ MFSB is the abbreviation of multi-fine-shear-band.

$\ddagger$ The gauge length of this specimen is $3 \mathrm{~mm}$. 

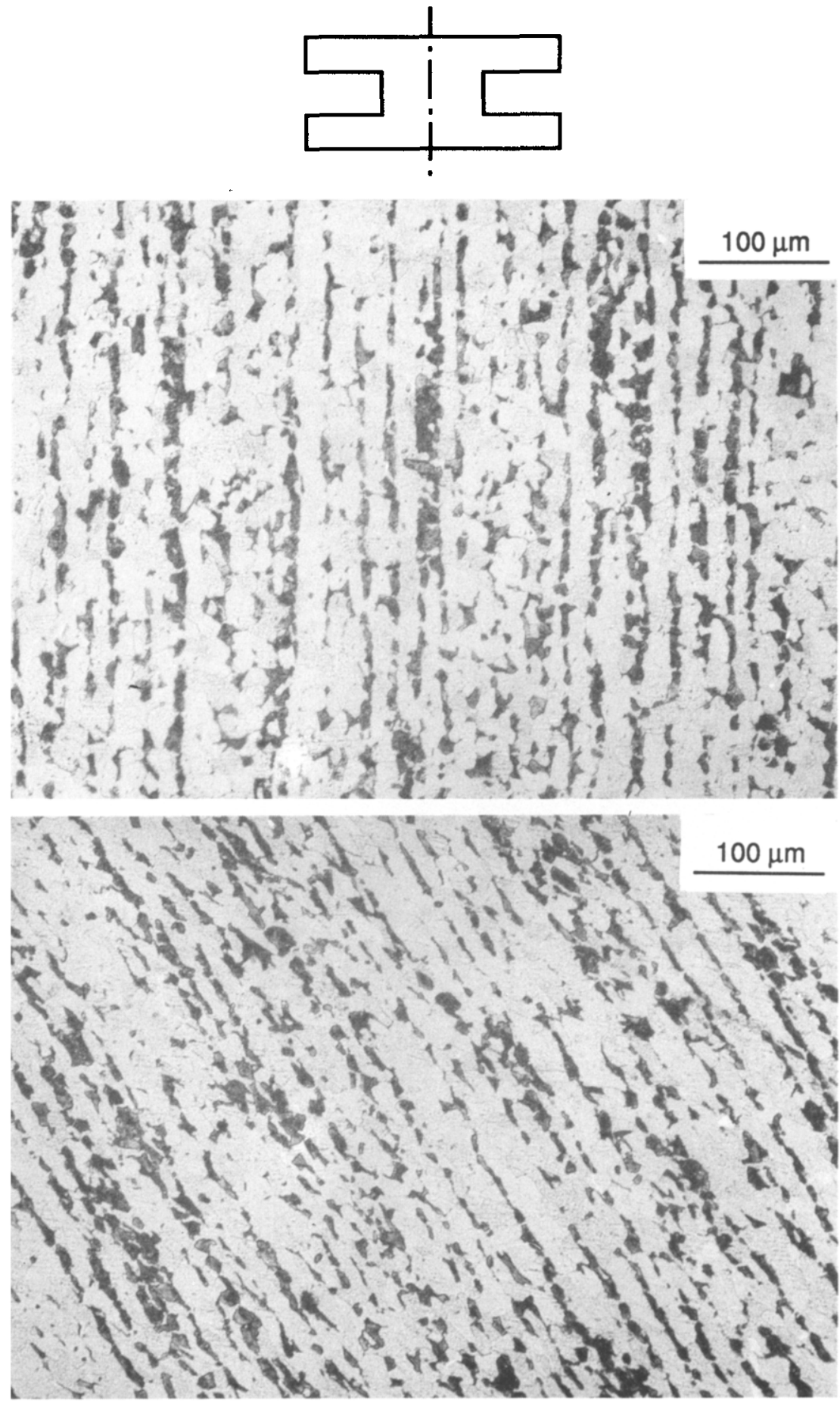

Fig. 2. Texture of specimen. (a) Undeformed specimen, (b) uniformly deformed specimen. 


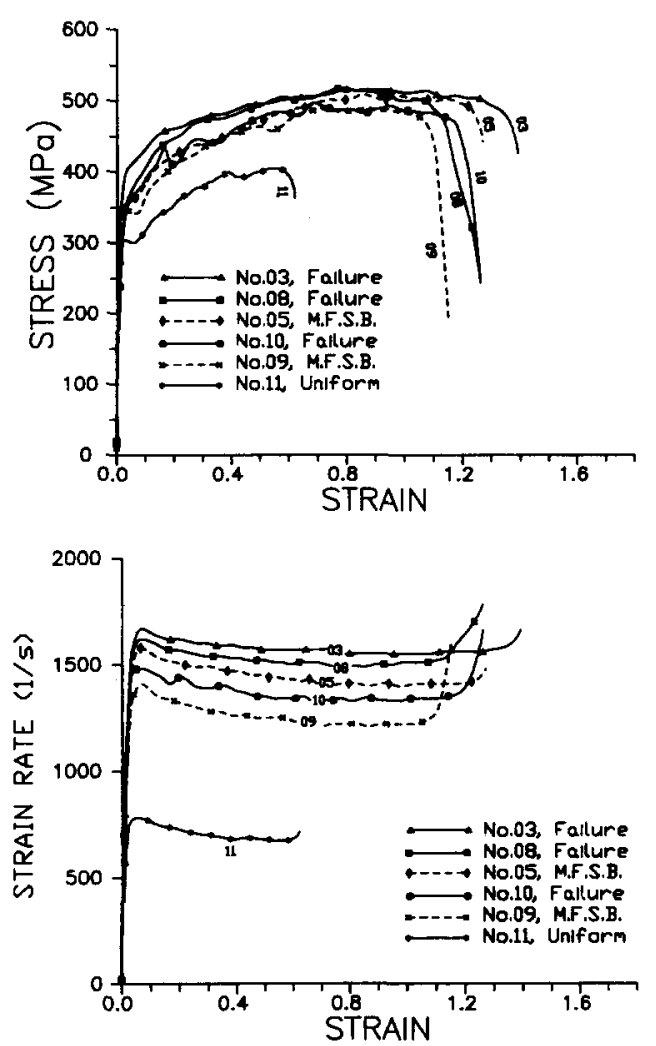

FIG. 3. Stress-strain and strain rate-strain curves. (a) $\tau-\gamma$ curves, (b) $\dot{\gamma}-\gamma$ curves.

2. The final shear localization occurs at a nominal strain of about $1.25-1.40$, when the stress drops severely and fracture occurs. Only one fully developed shear band is observed.

3. The multi-fine-shear-band phenomenon was observed, from metallography, at a nominal strain from 0.94 to 1.13 . Assuming that unloading acts uniformly on the specimen due to the very short gauge length and high sound speed of the specimens, we calculate (using the recorded incident and transmitted pulses) that the nominal strain during the test for this phenomenon is about 1.1-1.26. This is in the range between the maximum of the stress-strain curve and the occurrence of ultimate shear localization. Experimental evidence for uniform unloading is given in the metallograph of Fig. 2b, which was deformed at a strain rate of about $700 \mathrm{~s}^{-1}$ on the same instrument.

\section{Numerical Simulation}

A code making use of the Green's function method and numerical integration was developed to study thermo-plastic instability in simple shear and the subsequent processes of shear banding due to FADs. 


\subsection{Governing equations}

As shown in Fig. 6, a block of length $L$ is deformed by a constant driving velocity $V_{0}$ under adiabatic boundary conditions. The initial conditions are that the specimen is homogeneous, at room temperature and in a strain free state.

The equations listed below which govern the specimen deformation were first published by BAI (1982) (the overbar means the variables are dimensional). Inertial and elastic effects can be neglected for the process of shear banding (BAI, 1989). For deformation that is homogeneous on the scale of the specimen, the shear stress $\tau$ is a function of time only $(\bar{\tau}=\bar{\tau}(\bar{t}))$, so that

$$
\frac{\partial \bar{\tau}}{\partial \bar{x}}=0
$$

Plastic deformation acts as a source of heat, and the rate of generation of heat is some fraction $\beta$ of the plastic work rate $\bar{\tau} \bar{\gamma}[\beta$ is usually taken to be 0.9 after TAYLOR and QUINNEY (1934)]. Thus the one-dimensional heat diffusion equation becomes

$$
\rho c \frac{\partial \bar{\theta}}{\partial \bar{t}}=\beta \bar{\tau} \bar{\gamma}+k \frac{\partial^{2} \bar{\theta}}{\partial \bar{x}^{2}} .
$$

In order to compare our numerical simulation with the results of MOLINARI and CLIfTON (1987), we adopted their constitutive equation for HRS

$$
\bar{\tau}=\bar{\tau}(\bar{\gamma}, \gamma, \bar{\theta})=\mu \bar{\gamma}^{m} \gamma^{n} \bar{\theta}^{\prime} .
$$

The boundary conditions we adopted were as follows: we assume the deformation is rapid enough that no heat flows out through the specimen ends (adiabatic deformation). Thus

$$
\left.\begin{gathered}
\partial \bar{\theta} \\
\partial \bar{x}
\end{gathered}\right|_{0}=\left.\begin{aligned}
& \partial \bar{\theta} \\
& \partial \bar{x}
\end{aligned}\right|_{l}=0 .
$$

Since the specimen is clamped at $\bar{x}=0$ and constrained to move at a velocity $V_{0}$ at $\bar{x}=L$,

$$
\int_{0}^{1} \bar{\gamma}(\bar{x}, \bar{t}) \mathrm{d} x=V_{0}
$$

must be true. Also the specimen is initially unstrained at $\bar{t}=0$

$$
\gamma(\bar{x}, 0)=0 .
$$

The initial strain rate at $\bar{t}=0$ is

$$
\bar{\gamma}(x, 0)=\dot{\gamma}_{0}=V_{0} / L
$$

and the initial temperature $\bar{\theta}_{c}$ in the specimen is the same everywhere

$$
\bar{\theta}(\bar{x}, 0)=\bar{\theta}_{\epsilon} .
$$

We may define nondimensional quantities as follows : 


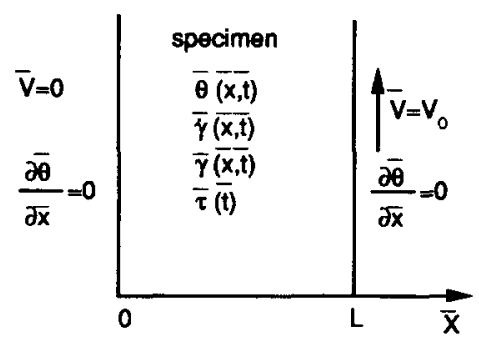

FIG. 6. Schematic diagram of deformed region $0<x<L$.

$$
\begin{aligned}
& x=\bar{x} / \Delta, \quad t=\dot{\gamma}_{0} \bar{t}=\gamma_{\text {nominal }}, \quad \tau=\bar{\tau} / \tau_{b}, \quad \theta=\bar{\theta} / \theta_{0}, \\
& P=\frac{k}{\rho c \Delta^{2} \dot{\gamma}_{0}}, \quad R=\frac{\beta \tau_{b}}{\rho c \theta_{0}}, \quad A=\frac{\mu \dot{\gamma}_{0}^{m} \theta_{0}^{v}}{\tau_{b}}, \quad a=L / \Delta .
\end{aligned}
$$

Here $\tau_{b}$ is the characteristic shear strength, $\theta_{0}$ is the characteristic temperature rise and $\Delta$ is the characteristic width of a shear band. In this paper, we take $\tau_{b}=100 \mathrm{MPa}$, $\theta_{0}=100^{\circ} \mathrm{C}, \Delta=0.4 \mathrm{~mm}$ (XU et al., 1989). Equations (1)-(8) may be normalized by substituting the above relations. Thus we obtain

$$
\begin{gathered}
\tau=\tau(t), \\
\frac{\partial \theta}{\partial t}=R \tau \dot{\gamma}+P \frac{\partial^{2} \theta}{\partial x^{2}}, \\
\tau=\tau(\dot{\gamma}, \gamma, \theta)=A \dot{\gamma}^{m} \gamma^{n} \theta^{v}, \\
\left.\frac{\partial \theta}{\partial x}\right|_{0}=\left.\frac{\partial \theta}{\partial x}\right|_{a}=0, \\
\frac{1}{a} \int_{0}^{a} \dot{\gamma}(x, t) \mathrm{d} x=1, \\
\gamma(x, 0)=0, \\
\dot{\gamma}(x, 0)=1, \\
\theta(x, 0)=\theta_{e} .
\end{gathered}
$$

\subsection{Numerical methodology}

In phase I $\left(0<t<t_{s}\right)$, we suppose that the specimen deforms uniformly with the temperature rising homogeneously. $\dot{\gamma}, \gamma, \theta$ and $\tau$ at this stage can be found analytically.

At $t=t_{s}$, a number of FADs are introduced into the distribution of strain rate and temperature. Each disturbance is in the form of a Gauss function, distinguished by its position, half width and disturbing energy (expressed as a percentage of the total thermal energy).

In phase II $\left(t_{s}<t<t_{e}\right)$, numerical calculations are carried out to simulate the process in which FADs evolve into shear bands. A Green's function for the adiabatic 
boundary and numerical integration were used in this phase. Details of the numerical methodology are given below.

3.2.1. For $0<\mathrm{t}<\mathrm{t}_{\mathrm{s}}$. The specimen is supposed to deform homogeneously and the temperature rises homogeneously too, i.e.

$$
\frac{\partial \theta}{\partial x}=\frac{\partial \gamma}{\partial x}=\frac{\partial j}{\partial x}=0
$$

The governing equations at this stage are

$$
\begin{gathered}
\frac{\mathrm{d} \theta}{\mathrm{d} t}=R \tau \dot{\gamma}, \\
\tau=A \dot{\gamma}^{m} \gamma^{n} \theta^{v}, \\
\gamma=\dot{\gamma}_{0} t, \\
\dot{\gamma}=\dot{\gamma}_{0}=1, \\
\left.\theta\right|_{t=0}=\theta_{e}+2.73 .
\end{gathered}
$$

Integrating these equations with respect to time, we obtain

$$
\begin{gathered}
\theta=\left[\frac{1-v}{1+n} R A t^{n+1}+\left(\theta_{e}+2.73\right)^{1-v}\right]^{1: 1-v}-2.73, \\
\tau=A t^{n} \theta^{v}, \\
\gamma=t, \\
\gamma=\dot{\gamma}_{0}=1 .
\end{gathered}
$$

Here $\theta_{e}$ represents the nondimensional temperature of the environment.

3.2.2. For $\mathrm{t}=\mathrm{t}_{\mathrm{s}}$. At this time, disturbances of finite amplitude in temperature were introduced into the homogeneous background (Fig. 7). After the disturbance, the temperature distribution was supposed to be

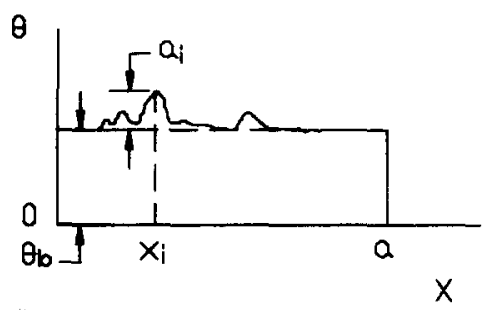

Fig. 7. Schematic diagram of disturbed temperature distribution 


$$
\theta\left(x, t_{s}^{+}\right)=\theta_{b}+\sum_{i=1}^{N} a_{i} f_{i}(x),
$$

where $N$ denotes the number of disturbances, $f_{i}(x)$ represents the distribution function and $a_{i}$ the magnitude of the $i$ th FAD. In this paper, $f_{i}(x)$ is a Gauss function

$$
f_{i}(x)=\exp \left[-\frac{\left(x-x_{i}\right)^{2}}{\Delta x_{i}^{2}}\right]
$$

where $\Delta x_{i}$ is the characteristic half width and $x_{i}$ is the location of the $i$ th FAD. It is supposed that $2 \Delta x_{i} \ll a$ (i.e. disturbances have a short wavelength). Therefore, we have

$$
\int_{0}^{a} f_{i}(x) \mathrm{d} x=\int_{-\infty}^{+\infty} \exp \left[-\frac{\left(x-x_{i}\right)^{2}}{\Delta x_{i}^{2}}\right] \mathrm{d} x=\sqrt{ }(\pi) \Delta x_{i} .
$$

If the time interval between $t_{s}^{-}$and $t_{s}^{+}$is so small that:

(a) the strain state of the block in Fig. 6 remains unchanged,

(b) the total energy of the block does not change,

then along with the following assumptions that :

(c) elastic and inertial effects can be neglected,

(d) the constant velocity and adiabatic boundary conditions remain true,

we have

$$
\begin{gathered}
\gamma\left(x, t_{s}^{-}\right)=\gamma\left(x, t_{s}^{+}\right), \\
\int_{0}^{a} \theta\left(x, t_{s}^{-}\right) \mathrm{d} x=\int_{0}^{a} \theta\left(x, t_{s}^{+}\right) \mathrm{d} x, \\
\int_{0}^{a} \dot{\gamma}\left(x, t_{s}^{-}\right) \mathrm{d} x=\int_{0}^{a} \dot{\gamma}\left(x, t_{s}^{+}\right) \mathrm{d} x, \\
\left.\frac{\partial \theta}{\partial t}\right|_{0}=\left.\frac{\partial \theta}{\partial t}\right|_{a}=0, \\
\tau=A \dot{\gamma}^{m} \gamma^{n} \theta^{v} .
\end{gathered}
$$

Thus, each FAD in temperature is distinguished by its position $x_{i}$, its half width $\Delta x_{i}$, and the percentage concentration energy $E_{i}$. Here $E_{i}$ is defined as

$$
E_{i}=\frac{\text { energy concentrated in the } i \text { th FAD }}{\text { total energy (thermal) }}=\frac{a_{i} \int_{0}^{a} f_{i}(x) \mathrm{d} x}{\int_{0}^{a} \theta\left(x, t_{s}\right) \mathrm{d} x} .
$$

By solving Eqs (27)-(35), we obtain 
1030

$$
\begin{gathered}
a_{i}=\left[E_{i} \int_{0}^{a} \theta\left(x, t_{s}^{-}\right) \mathrm{d} x\right] /\left(\sqrt{ }(\pi) \Delta x_{i}\right), \\
\theta_{b}=\frac{1}{a}\left(1-\sum_{i=1}^{N} E_{i}\right) \int_{0}^{a} \theta\left(x, t_{s}\right) \mathrm{d} x \\
\theta\left(x, t_{s}^{+}\right)=\theta_{b}+\sum_{i=1}^{N} a_{i} f_{i}(x) .
\end{gathered}
$$

From Eq. (34) and $\theta\left(x, t_{s}^{+}\right)$obtained in Eq. (38), $\gamma, \dot{\gamma}$ and $\tau$ at $t_{s}^{+}$can be found analytically as

$$
\begin{gathered}
\gamma\left(x, t_{s}\right)=\gamma\left(x, t_{s}^{+}\right), \\
\tau^{1 / m}=\frac{a}{\left.\int_{a}^{a}\left(A \gamma^{n} \theta^{v}\right)^{-1 / m} \mathrm{~d} x\right|_{t=t_{s}^{+}},} \\
\dot{\gamma}=\left.\left[\frac{\tau}{A \gamma^{n} \theta^{v}}\right]^{1 / m}\right|_{l=t_{s}^{+}} .
\end{gathered}
$$

Now, it is very clear that, for a given $N, E_{i}, x_{i}$ and $\Delta x_{i}$, we may find the disturbed $\theta\left(x, t_{s}^{+}\right), \dot{\gamma}\left(x, t_{s}^{+}\right), \gamma\left(x, t_{s}^{+}\right)$and $\tau\left(t_{s}^{+}\right)$from Eqs $(36)-(41)$.

3.2.3. For $\mathrm{t}_{\mathrm{s}}<\mathrm{t}<\mathrm{t}_{\mathrm{c}}$. At this stage, a numerical calculation is carried out, based on Eqs (9)-(16), to simulate the process in which FADs evolve into a shear band. Numerical integration and a Green's function for an adiabatic boundary are used. Here, Eq. (10) is rewritten as

$$
\frac{\partial \theta}{\partial t}=R Q+P \frac{\partial^{2} \theta}{\partial x^{2}}
$$

where $Q=\tau \dot{\gamma}$, subject to the same boundaries condition and constitutive relations as before.

3.2.4. Integral method. The temperature $\theta$ at $\left(x, t_{n}\right)$ in Eq. (42) can be solved within the region shown in Fig. 8 as

$$
\theta\left(x, t_{n}\right)=\iint_{\Omega} R Q G\left(\xi, \eta ; x, t_{n}\right) \mathrm{d} \xi \mathrm{d} \eta+\int_{0}^{a} \theta\left(\xi, t_{n} \quad 1\right) G\left(\xi, t_{n, 1}, x, t_{n}\right) \mathrm{d} \xi
$$

where $G\left(\xi, \eta ; x, t_{n}\right)$, the Green's function for an adiabatic boundary, is determined by 


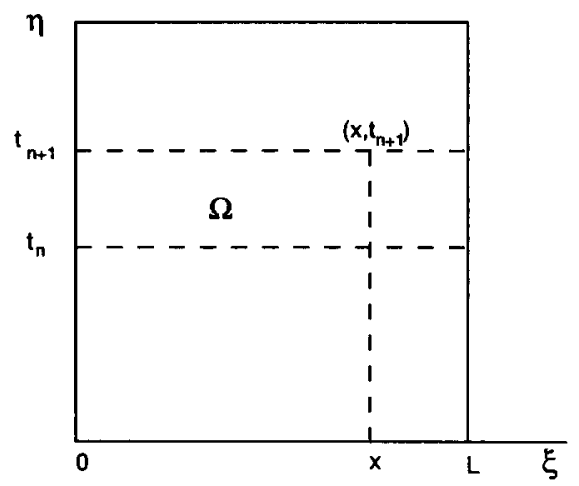

FIG. 8. Integral domain of Eq. (42).

$$
\frac{\partial G}{\partial \eta}+P \frac{\partial^{2} G}{\partial \xi^{2}}=\delta(\xi-x) \delta\left(\eta-t_{n}\right)
$$

subject to the boundary conditions

$$
\left.\frac{\partial G}{\partial \xi}\right|_{0}=\left.\frac{\partial G}{\partial \xi}\right|_{a}=0,\left.\quad G\right|_{t_{n}}=0
$$

Integrating Eq. (44) over $t_{n}^{-}<\eta<t_{n}^{+}$, we obtain

$$
\begin{gathered}
\frac{\partial G}{\partial \eta}+P \frac{\partial^{2} G}{\partial \xi^{2}}=0 \\
\left.\frac{\partial G}{\partial \xi}\right|_{0}=\left.\frac{\partial G}{\partial \xi}\right|_{a}=0,\left.\quad G\right|_{\eta=t_{n}}=\delta(\xi-x) .
\end{gathered}
$$

Thus, we have

$$
G\left(\xi, \eta ; x, t_{n}\right)=\frac{2}{a} \sum_{n=0}^{\infty} \exp \left[k_{n} P\left(\eta-t_{n}\right)\right] \cos \left(k_{n} x\right) \cos \left(k_{n} \xi\right)
$$

where $k_{n}=n \pi / a, n=0,1,2, \ldots$ From Eq. (47), using trapezoidal integration over $t_{n-1}<\eta<t_{n}$, Eq. (43) becomes

$$
\begin{aligned}
& \theta\left(x, t_{n}\right)=\int_{0}^{a}\left[\frac{t_{n}-t_{n-1}}{2} R\right. R\left(\xi, t_{n-1}\right) \\
&\left.+\theta\left(\xi, t_{n-1}\right)\right] G\left(\xi, t_{n-1} ; x, t_{n}\right) \mathrm{d} \xi+R Q\left(x, t_{n}\right) \frac{t_{n}-t_{n-1}}{2} .
\end{aligned}
$$

Equation (49) is a very important formula, because it shows that the temperature distribution $\theta\left(x, t_{n}\right)$ is determined by the previous temperature $\theta\left(x, t_{n-1}\right)$, the intensity distribution of previous heating point $Q\left(\xi, t_{n-1}\right)$ and the intensity of present heating $Q\left(x, t_{n}\right)$, while $Q\left(x, t_{n}\right)=\tau\left(x, t_{n}\right) \gamma\left(x, t_{n}\right)$ is solved by Eqs $(9),(11)-(15)$. 


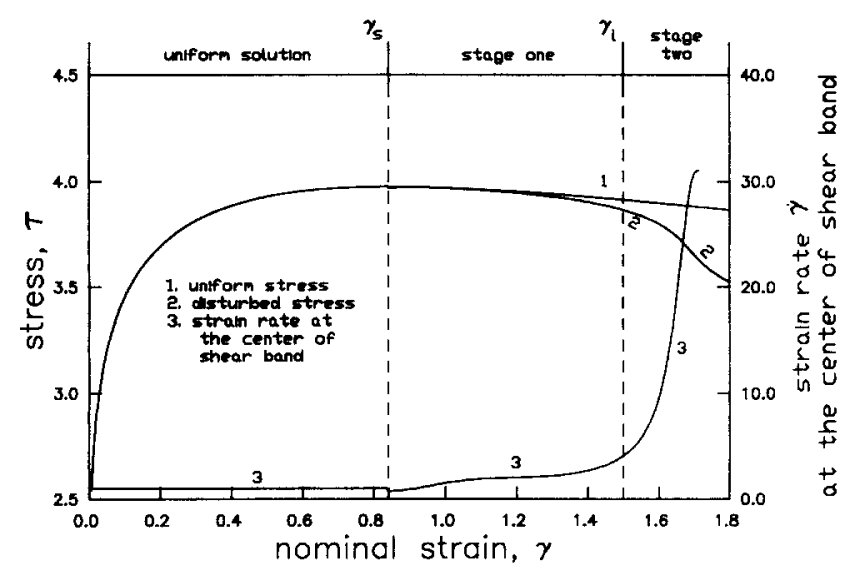

Fig. 9. Stress, strain rate vs nominal strain.

\subsection{Numerical simulation}

To investigate the shear banding process in detail, a number of numerical simulations were carried out. In running the code for HRS, the material parameters were taken as:

$$
\begin{aligned}
& V_{0}=3.0 \mathrm{~m} / \mathrm{s}, \quad L=2.0 \mathrm{~mm}, \\
& \dot{\gamma}_{0}=1500 \mathrm{~s}^{-1} \\
& \rho=7.82 \times 10^{3} \mathrm{~kg} / \mathrm{m}^{3}, \quad c=4.73 \times 10^{2} \mathrm{~J} / \mathrm{kg}{ }^{\circ} \mathrm{C}, \quad k=0.494 \times 10^{2} \mathrm{~J} / \mathrm{s} \mathrm{m}^{\circ} \mathrm{C} \\
& \mu=7587 \times 10^{6} \text { S.I. }, \quad m=0.0133, \quad n=0.12, \quad v=0.51 \text {. }
\end{aligned}
$$

Parameters $\mu, m, n$ and $v$ for HRS were given by MolinARI and CLIFTON (1987).

Our calculation shows that there is very little effect on the features of the shear banding process (including the width of the final developed shear band) of using three different kinds of mesh size, i.e. 100, 150, and 200 divisions in the block (Fig. 6). The differences in $\gamma_{l}$ (defined in next paragraph) are less than $3 \%$ for these three meshes. In this paper, all calculations are carried out using a mesh of 200 divisions.

Figure 9 shows the relationship of the dimensionless stress and local shear strain rate at the centre of the shear band vs nominal shear strain $\gamma$. Line 1 stands for the uniform analytic solution of quasi-static approximations which gives a strain of 0.84 for the maximum. This is in good agreement with the experimental result $\gamma_{c}$ between 0.65 and 0.88 (Table 2). Line 2 represents the disturbed stress with four equal-inamplitude FADs, which were applied at the stress maximum $\gamma_{c}=0.84$. Table 3 gives the parameters of the four FADs and the calculated $\gamma_{l}$. Here $\gamma_{l}$ is a nominal strain for localization and is defined to be the point at which the disturbed stress drops $5 \%$ from the uniform analytical solution. Line 3 in Fig. 9 is the development of disturbed

TABLF 3

\begin{tabular}{ccccc}
\hline$E$ & $E_{\text {total }}$ & $\Delta x$ & $\gamma_{s}$ & $\gamma_{t}$ \\
\hline $0.5 \%$ & $2.0 \%$ & 0.1 & 0.84 & 1.50 \\
\hline
\end{tabular}


strain rate. It is clearly shown that, before $\gamma=\gamma_{l}=1.50$, the stress evolves close to the uniform solution and the strain rate at the centre of the shear band grows slowly. But after $\gamma>\gamma_{l}=1.50$, a drastic drop in the stress and a sharp increase in the local shear strain rate occur simultaneously. As pointed out by MARCHAND and DUFFY (1987), the sharp drop in stress during an experiment is due to cracking. But in our calculation, cracking effects are not considered. So the stress drop in Fig. 9 is not as sharp as in those experiments.

To simulate a number of fine shear bands appearing near the experimentally observed stress peak, up to five FADs with a total energy ranging from $0.1 \%$ to $3.0 \%$ were adopted in computation. All cases show similar late-stage behaviour, namely a single shear band appearing in the deformed region. A typical evolution is shown in Fig. 10a-c. There, four FADs gradually merge into a contracting localized area at about $\gamma=\gamma_{l}=1.50$, where a drastic decline of the disturbed stress occurs. Afterwards, as the shear strain rate and the temperature of the shear band increase rapidly, the band shrinks. Finally, the ultimate shear band is formed, showing a quite stable band width, although the temperature continuously diffuses. The width of the final shear band is found to be independent of the FADs chosen.

\subsection{Discussion of the numerical simulation}

3.4.1. Two stages from the application of FADs to the final shear band. A detailed study of the process of shear banding reveals that there seems to be two distinct stages, separated by $\gamma_{l}$ (see Fig. 9). During the first stage $\left(\gamma<\gamma_{l}\right)$, the stress evolves close to

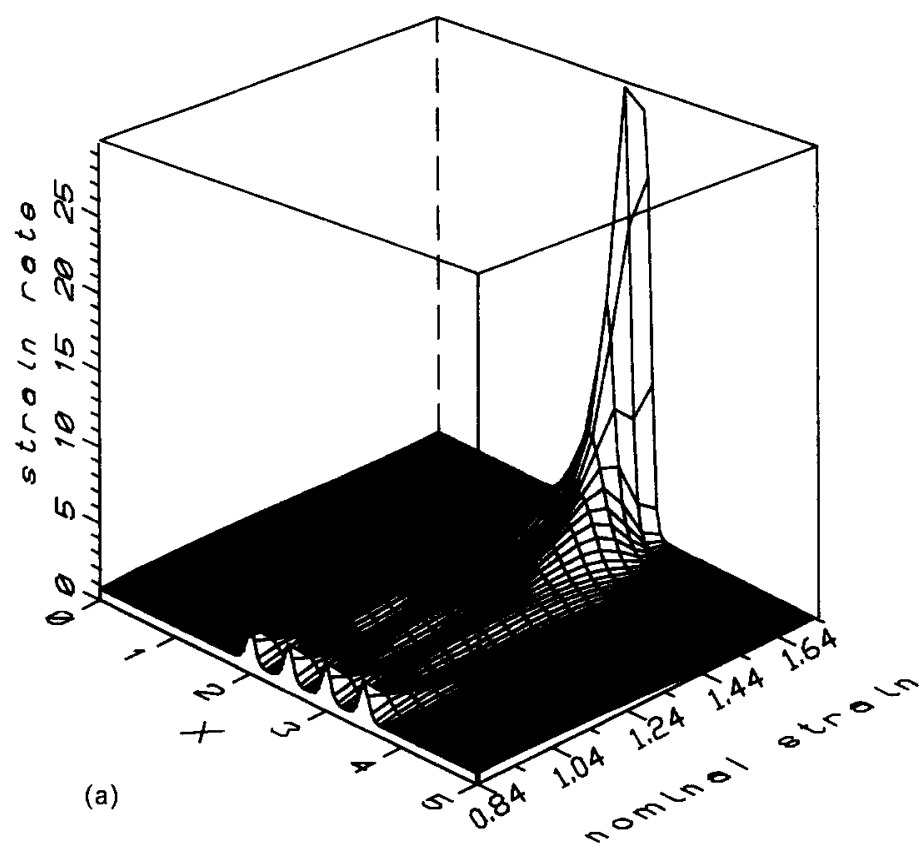

FIG. 10(a). 

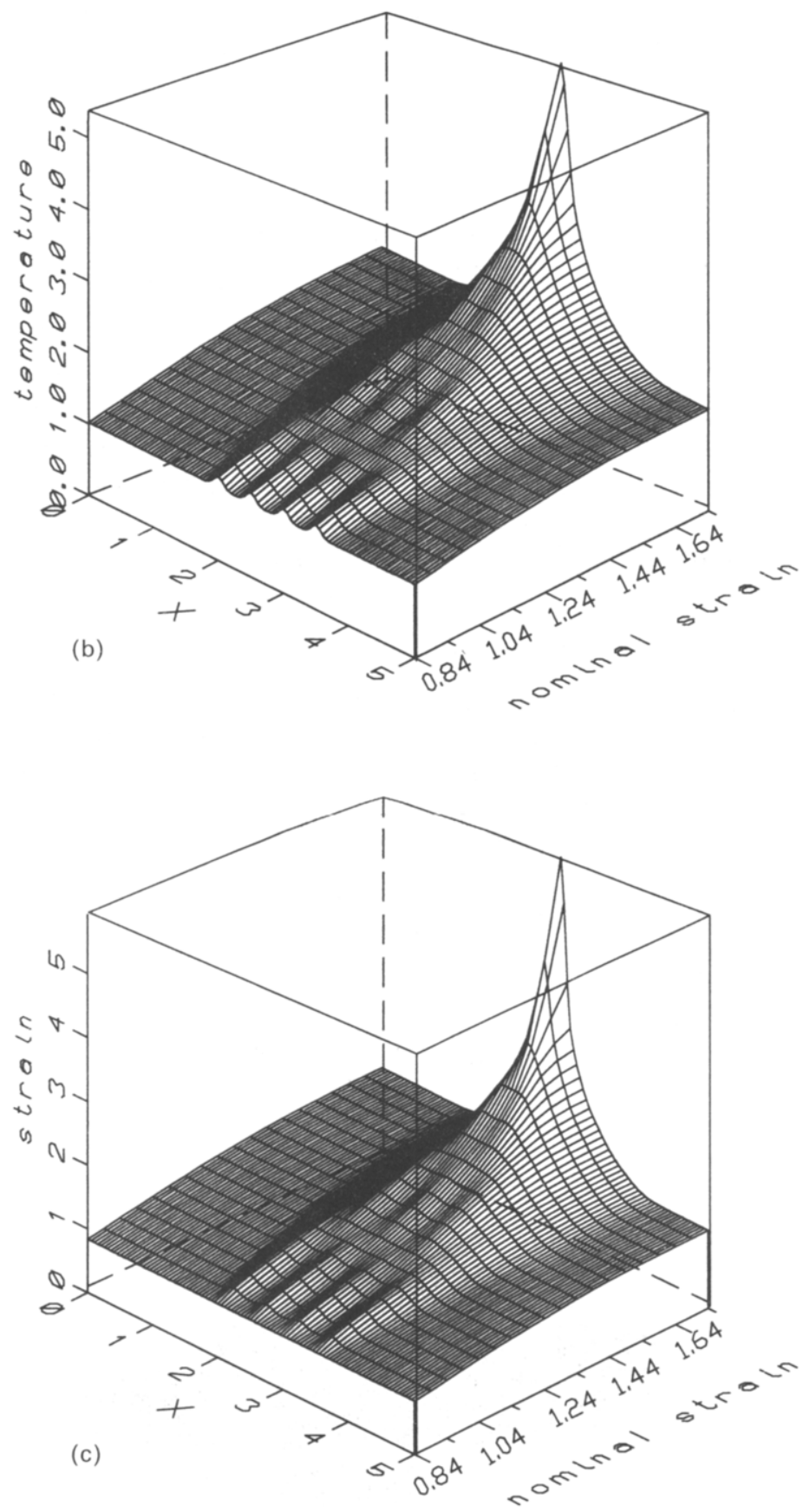

IG. 10. Evolution of one shear band from four FADs. (a) Strain rate evolution, (b) temperature evolu (c) strain evolution. 
that of the uniform solution (Fig. 9) and the FADs coalesce into one local area (Fig. 11), whereas during the second stage $\left(\gamma>\gamma_{l}\right)$, severe localization occurs, the shear band shrinks to its final form (Fig. 12), the stress drops drastically and the strain rate at the centre of shear band increases sharply (Fig. 9). Therefore, the first stage can be denoted the coalescence stage and the second stage to be the localization stage.

3.4.2. The effect of FADs on the shear banding process. Numerical simulations show that different FADs give different coalescence behaviour but similar localization. $\gamma_{l}$ is an indicator for the occurrence of localization. The smaller the $\gamma_{l}$ is, the earlier the localization occurs. This section investigates the effects of different aspects of FADs on $\gamma_{l}$.

In Fig. 13, three kinds of FADs are applied at different disturbing strains $\gamma_{s}$ ranging from 0.22 to 1.2 , the relationships of their localized strain $\gamma_{l}$ and disturbing strain $\gamma_{s}$ are given in the upper three curves. The horizontal line at the bottom of this figure is a reference line, representing a strain value of 0.84 (the critical strain $\gamma_{c}$ at the maximum of the stress curves), obtained from infinitesimal perturbation analysis. The fact that the three curves in Fig. 13 are superior to the $\gamma_{c}=0.84$ reference line means, for these kinds of FADs, that $\gamma_{l}>\gamma_{c}$, so we can say the critical strain $\gamma_{c}$ is the lower bound for increasing inhomogeneous shear, i.e. no shear band occurs before $\gamma_{c}$. Figure 13 also shows that the FADs imposed in the vicinity of $\gamma_{c}$ lead to the lowest value of $\gamma_{l}$. So in all examples below, we take $\gamma_{s}=\gamma_{c}$.

The effect of the wavelength is shown in Fig. 14 which indicates that disturbances of short wavelength give low values of $\gamma_{1}$, due to the faster growth rate of short wavelength disturbances compared to that of long wavelength disturbances. This result is in accordance with the statement, put forward by CLIFTON (1984) and BAI (1982), that disturbances of short wavelength grow first.

Figure 15 discusses the effects of disturbance energy to the value of $\gamma_{1}$. One can see that as the disturbance energy $E$ increases, the value of $\gamma_{l}$ decreases. Disturbances with low energy $(0.2-1.0 \%)$ decrease faster than those with high energy $(2.0-3.0 \%)$. All these results suggest the importance of investigating the effects of short wavelength disturbances with low disturbance energy on adiabatic shear banding after reaching the maximum of the stress-strain curve. It is worth noting that, in Fig. 15, for $E<0.1 \%$ no $\gamma_{l}<2.0$ occurred. This suggests the importance of FADs with a certain amount of energy on localization in finite loading time.

Furthermore, the effects of the distribution of FADs on the site and time $\left(\gamma_{l}\right)$ of localization were studied.

The site of localization, i.e. the position of the final developed single shear band, is found to depend on the distribution of FADs, but does not correspond to any individual FAD. A typical result is shown in Fig. 16. There, two FADs with total energy of $1 \%$ were applied initially at $\gamma_{s}=0.84$ at $x=2$ and 3, respectively. Thróugh a very complicated evolution process, the final developed shear band appeared at $x=2.5$, which is not the position of any individual FAD. This is qualitatively consistent with the experimental observations by SHOCKEY (1985). He discovered that the final shear band formed between two disturbed sites.

Five examples representing five kinds of FADs distribution are given in Table 4. There, FADs with total energy of $1 \%$ were added at $\gamma_{s}=\gamma_{c}=0.84$. The results in 

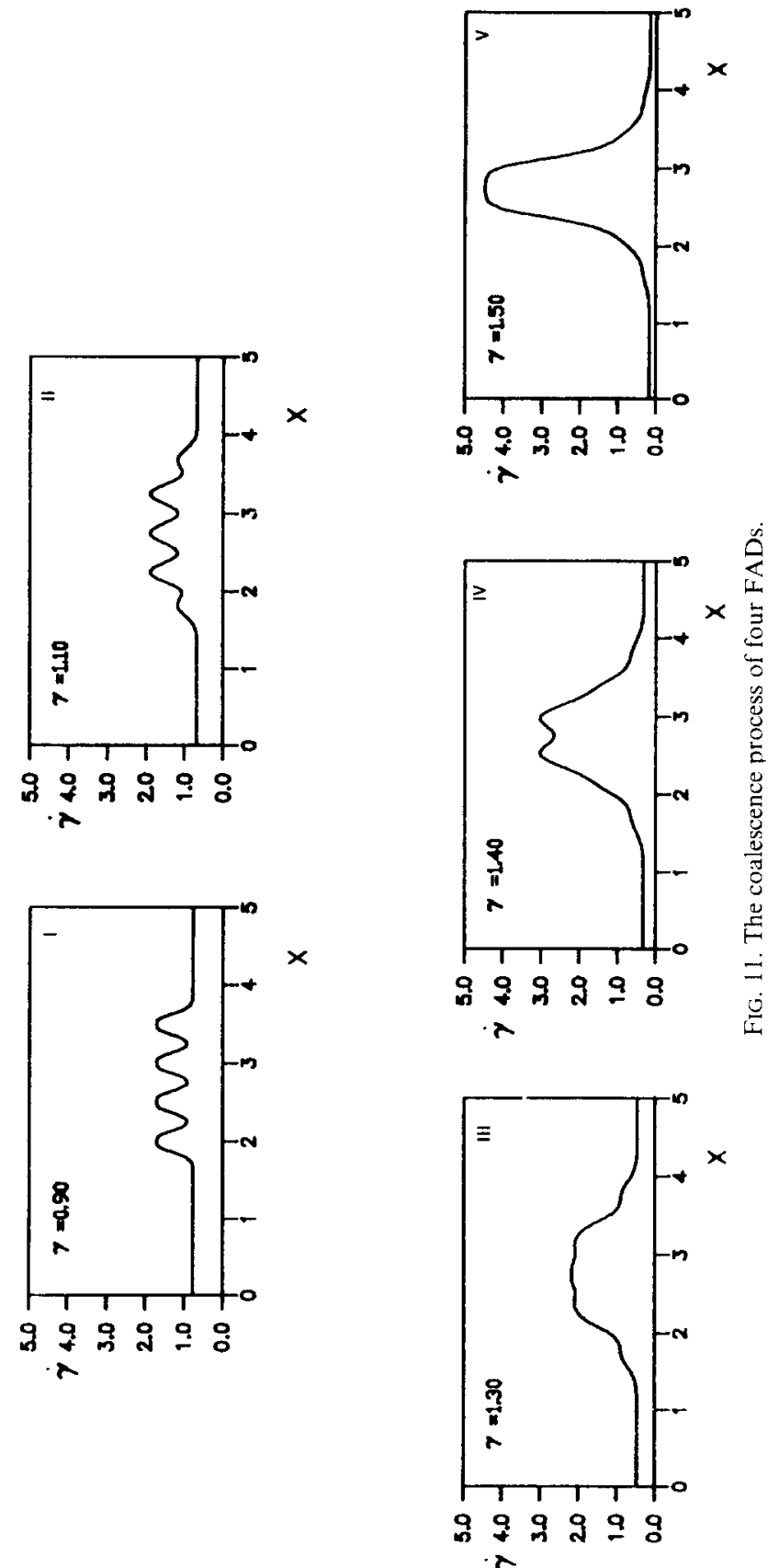


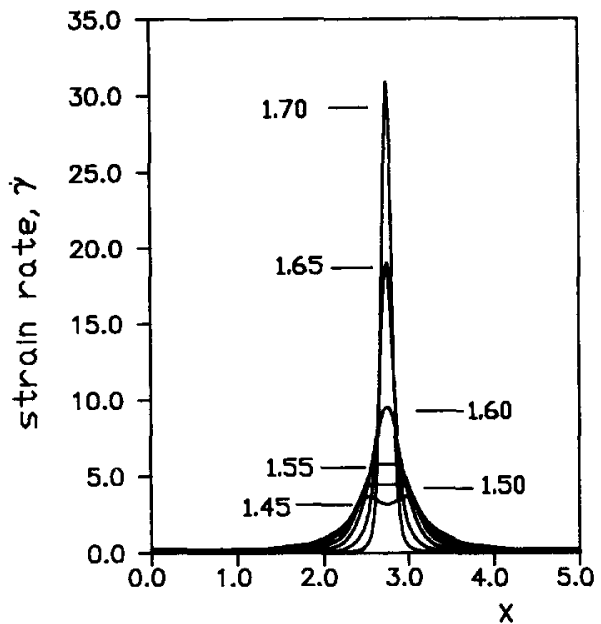

Fig. 12. Localization process at different nominal strain from 1.45 to 1.70 .

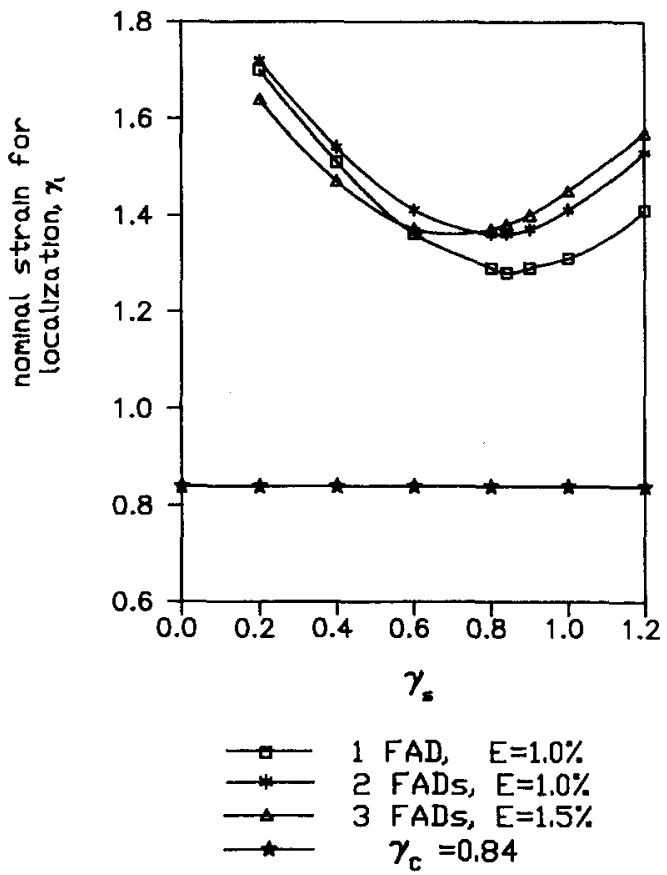

Fig. 13. $\gamma_{1}$ vs $\gamma_{s}$ 

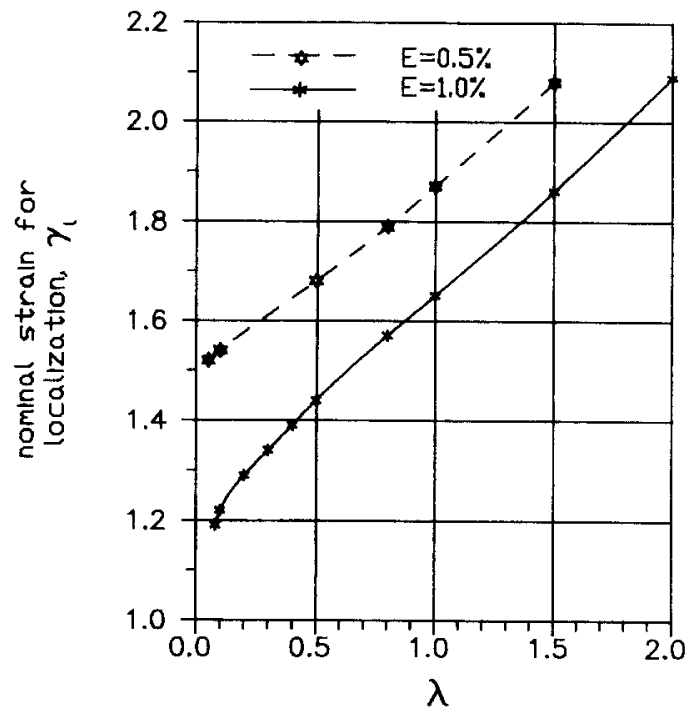

non-dimenslonal wave length

for a single disturbance

FIG. 14. The effect of wavelength $i$ on $i \frac{1}{}$

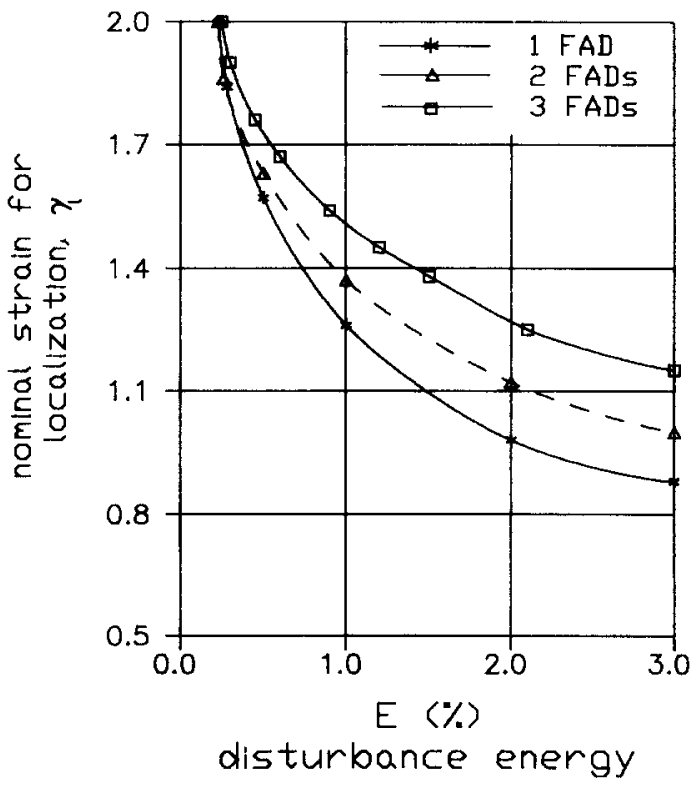

FIG. 15. The effect of disturbance energy $E$ on $\%$. 


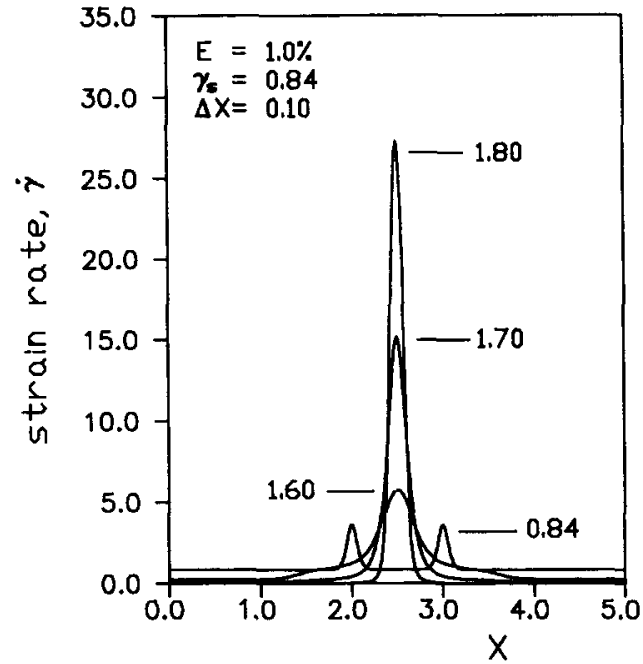

FIG. 16. The localization process of two FADs at nominal strain from 0.84 to 1.80 .

Table 4 show that different distribution gives different $\gamma_{l}$. This is understandable because different distributions show different coalescence processes. It is worth noting that the FAD distribution in example 5 shows very interesting features. The amplitudes of the five FADs are not identical, but give a contour of one single disturbance. This makes the FADs coalesce more easily and faster than that of examples 3 and 4 . The fact that $\gamma_{1}$ in example 5 is smaller than that of example 3 and 4 suggests that the easier the FADs coalescence, the smaller the value of $\gamma_{l}$ is, i.e. the earlier the localization occurs.

\section{SUMmary}

The process of shear banding was studied experimentally and numerically. The experiments showed that for the strain rate about $1270-1600 \mathrm{~s}^{-1}$ :

1. The nominal strain at stress maximum for 20 \# HRS is about $0.65-0.88$.

2. The final shear band occurred at nominal strain of 1.25-1.40. The width of this ultimate band is about $0.2-0.4 \mathrm{~mm}$.

3. Observations on the texture of two deformed specimens revealed multi-fineshear-bands over their gauge section and the texture also gives a nominal strain about 0.94-1.13. The reason for this phenomenon is not clear and the exact time for the occurrence of this phenomenon needs to be studied in further experiments.

Numerical simulation using a Green's function and numerical integration was devised to investigate the evolution of finite amplitude disturbances (FADs). Based on our numerical model, the critical shear strain $\gamma_{c}$ at the peak of the shear stress, obtained from infinitesimal perturbation analysis, seems to be a lower bound to 
TABLE 4. The effect of the FAD distribution on $\%$

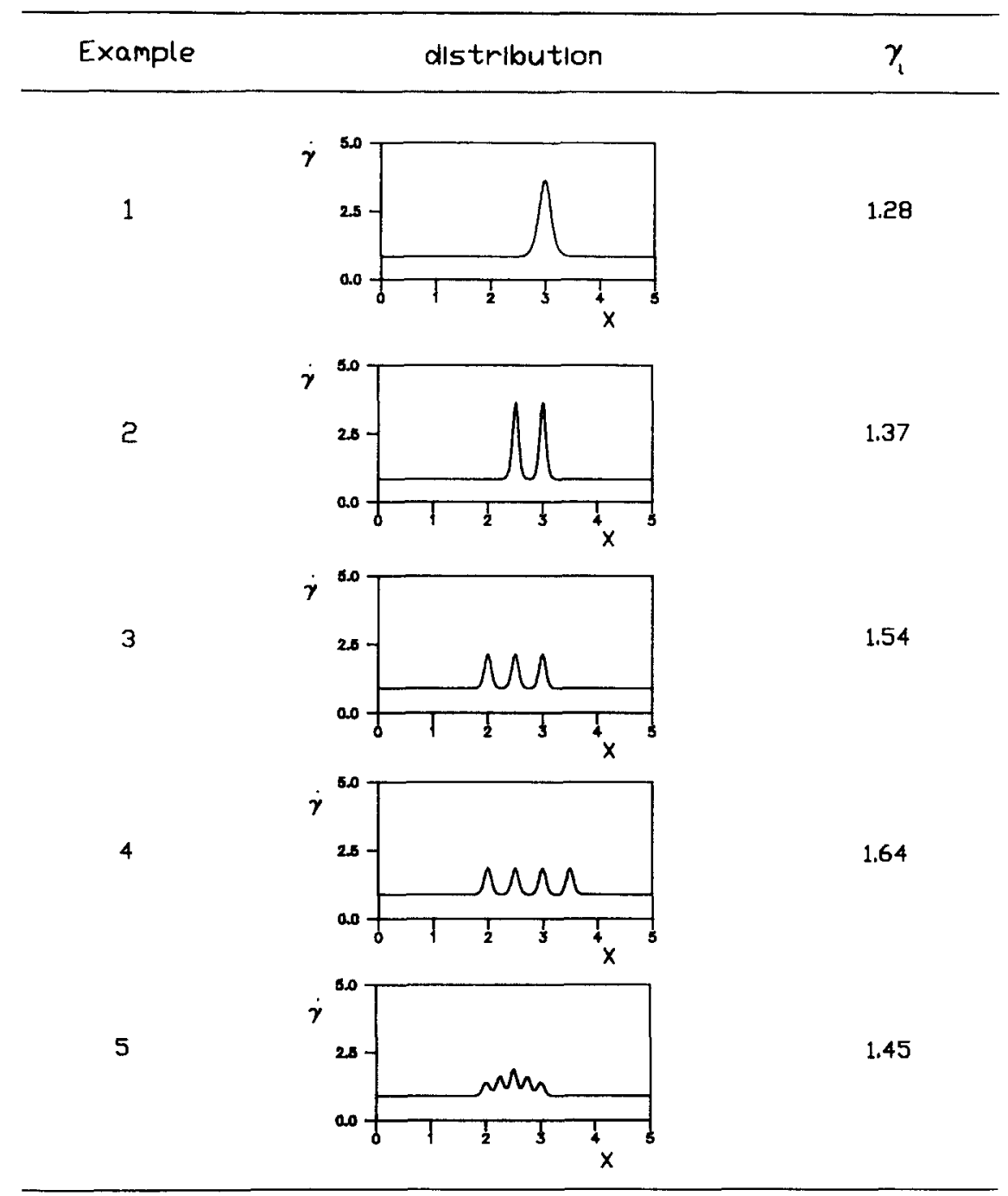

increasing inhomogeneous shearing. In the range of $0<\gamma<\gamma_{c}$, no shear localization occurs in the numerical simulations, even when some disturbances were imposed (as shown in Fig. 13). On the other hand, no shear localization appears until a nominal shear strain of 2.0, when disturbances with very low energy were applied (Fig. 15). Thus, FADs with a certain amount of disturbance energy are very important for the occurrence of severe localization in a limited loading time. As far as shear banding is concerned, there seem to be two distinct phases, separated by the localization strain $\gamma_{1}$. In the first stage $\left(\gamma<\gamma_{l}\right)$, the finite amplitude disturbances coalesce, and the stress evolves close to that of the uniform solution. Then in the second stage $\left(\gamma>\gamma_{l}\right)$, 
localization occurs, the shear band shrinks to its final form, the stress drops drastically and the strain rate at the centre of shear band increases sharply. This is the localization stage, in which a single shear band formed. This is qualitatively consistent with the experimental observations.

Furthermore, the effects of different aspects of finite amplitude disturbances on the evolution of shear bands were examined numerically. Firstly, the FADs imposed in the vicinity of $\gamma_{c}$ lead to the lowest value of localization strain $\gamma_{l}$. So do the disturbances with highest energy and shortest wavelength. Secondly, the position of the final developed shear band depends upon the distribution of FADs and not on any individual FAD. Finally, the distribution of FADs is found to have an important influence on the value of $\gamma_{l}$. Generally speaking, the easier the FADs coalesce, the smaller the $\gamma_{l}$ is, i.e. the earlier the localization occurs. It ought to be noted that all numerical results in this paper are dependent upon the constitutive equation given by MOLINARI and Clifton (1986).

\section{ACKNOWLEDGEMENT}

This work is jointly supported by Chinese Academy of Sciences under special grant No. 87-52 and National Natural Science Foundation of China. The authors would like to thank Dr S. M. Walley at Cavendish Laboratory for assistance in writing this paper.

\section{REFERENCES}

BAI, Y. L.

BAI, Y. L.

BAI, Y. L., ChENG, C. M. and YU, S. B.

BATRA, R. C.

Clifton, R. J., Duffy, J., HARTLEY, K. A. and SHAWKI, T. G.

Costin, L. S., Crisman, E. E., HAWLEY, R. H. and

DUFFY, $\mathbf{J}$

CULVER, R. S.

Drew, D. A. and Flaherty, J. E. 1984 Phase Transformations and Material Instabilities

Fressengeas, C. and MOLINARI, A

HARTLEY, K. A., DUFFY, J. and HAWLEY, R. H.

HarTLEY, K. A., DufFy, J. and HAWLEY, R. H.
1973 Metallurgical Effects of High Strain Rates (edited by R. W. Rohde, B. M. Butcher, J. R. Holland and C. H. KarNes), p. 519. Plenum, New York. in Solids (edited by GurTin MorTon). Aca-

1982 J. Mech. Phys. Solids 32, 195.

1989 4th Int. Conf. on Mech. Properties of Materials at High Rates of Strain, Oxford.

1986 Acta Mechanica Sinica 2, 1.

1987 Int. J. Solids Struct. 23, 1435.

1984 Scripta Metallurgica 18, 443.

1979 Inst. Phys. Conf. Ser. No. 47, 90.

$$
\text { demic Press, London. }
$$

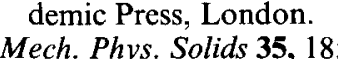

1987 J. Mech. Phys. Solids 35, 185.

1985 Metals Handbook, Vol. 8, p. 2163. American Society for Metals.

1987 J. Mech. Phys. Solids 35, 283. 
KWON, Y. W. and BATRA, R. C. MarChand, A. and DUFFY, J. Molinari, A. and Clifton, R. J.

ROGERS, R. C.

SHOCKEY, D. A.

STAKER, M. R.

TAYLOR, G. I. and QUINNEY, H.

WADA, $M$. and NAKamure, $T$.

WRIGHT, T. W. and WALTER, J. W.

XU, Y. B., WANG, Z. G.,

Huang, X. L., XING, D. and BAI, Y. L.

Zener, C. and Hollomon, J. H. 1944 J. Appl. Phys. 15, 22.
1988 Int. J. Engng Sci. 26, 1177.

1988 J. Mech. Phys. Solids 36, 251.

1987 Brown Univ. Tech. Report, ARO Gran DAAG29-85-K0003 Report No. 4.

1979 Ann. Rev. Mat. Sci. 9, 283.

1985 Poulter Lab. Tech. Report 004-85.

1980 Scripta Metallurgica 14, 677.

1934 Proc. R. Soc. Lond. A143, 307.

1978 Phil. Mag. A38, 167.

1987 J. Mech. Phys. Solids 35, 704.

1989 Mat. Sci. Engng A114, 81. 


\title{
DETERMINATION OF HIGHER-ORDER TERMS IN ASYMPTOTIC ELASTOPLASTIC CRACK TIP SOLUTIONS
}

\author{
S. M. Sharma and N. Aravas $\dagger$ \\ Department of Mechanical Engineering and Applied Mechanics, University of Pennsylvania, \\ Philadelphia, PA 19104, U.S.A.
}

(Received 11 June 1990)

\begin{abstract}
A METHODOLOGY for the calculation of higher-order terms in asymptotic elastoplastic crack tip solutions is developed. The $J_{2}$-deformation plasticity theory with power law hardening is used to describe the constitutive behavior of the continuum. A two-term expansion of the solution in the near crack tip region is developed. Plane stress and plane strain solutions for a crack in a homogeneous material as well as for a crack lying along the interface between a rigid substrate and an elastoplastic medium are obtained. For the case of a plane strain crack in a homogeneous material, it is shown that, when the hardening capacity of the material is small, the effects of elasticity enter the asymptotic solution to third order or higher; when there is substantial hardening, however, elastic effects enter the solution to second order and the magnitude of the second term in the expansion of the solution is controlled by the $J$-integral.
\end{abstract}

\section{INTRODUCTION}

THE CHARACTERIZATION of the stress and deformation fields in the region near the tip of a crack is essential for the development of sound fracture criteria. Hutchinson (1968) and RICE and RoSENGREN (1968) developed the elastoplastic asymptotic solution for the near-tip stresses in a homogeneous material (known as the HRR solution) and showed that the magnitude of the dominant term in the expansion of the solution is determined by the $J$-integral (RICE, 1968). If the region of dominance of the leading-order term in the expansion of the solution is sufficiently larger than the region over which the fracture micro-mechanisms take place, then the $J$-integral can be used as the fracture parameter. If the region of $J$-dominance, however, is smaller than the fracture process zone, then two or more parameters may enter the fracture criterion. Li and WANG (1986) suggested the use of a parameter $k_{2}$, which is the magnitude of the second term in the near-tip stress plastic solution, as the second parameter to be used together with the $J$-integral in the fracture criterion. BETEGón and HANCOCK (1991) used a modified boundary layer formulation of the small-scale yielding problem, in which the boundary conditions are defined in terms of the mode I stress intensity factor $K_{I}$ and the constant stress term $T$ that enters the near-tip expansion of the elastic solution (LARSSON and CARLSSON, 1973; RICE, 1974), and 
suggested $K_{I}$ and $T$ as an alternative set of parameters that can be used in the criterion for fracture initiation.

Li and WANG (1986) also presented a two-term expansion of the plastic solution near the tip of plane strain crack in a homogeneous material. They formulated the problem in terms of a stress function and presented numerical results for values of the hardening exponent $n=3$ and 10 . In this paper, we develop a methodology for the calculation of higher-order terms in two-dimensional asymptotic elastoplastic solutions near crack tips. The $J_{2}$-deformation plasticity theory with power law hardening is used to describe the constitutive behavior of the material. The problem is formulated in terms of fundamental quantities, namely stresses and displacements, thus avoiding the algebraic complications arising in the stress function approach. The formulation is quite general and it can be used for plane stress as well as plane strain problems. An asymptotic expansion of the solution in the near crack tip region is attempted, where both the first and the second terms in the stress expansion are assumed to be separable in $r$ and $\theta,(r, \theta)$ being polar coordinates at the crack tip. A hierarchy of problems is obtained, in which the leading-order problem consists of a set of five non-linear homogeneous ordinary differential equations that define the angular variation of the first-order stress and displacement components. The secondorder problem can be a linear eigenvalue problem or a set of non-homogeneous linear ordinary differential equations, depending on the value of the hardening exponent and the problem under consideration. Solutions are presented for the case of crack in a homogeneous material as well as for an interface crack with contact zones, the crack lying along the interface of an elastoplastic material and a rigid substrate. For the case of the plane strain crack in a homogeneous material, it is found that for large values of the hardening exponent $n$ (which corresponds to materials with small hardening capacity), the effects of elasticity enter the solution to third order or higher; in this case, the second-order problem is a linear eigenvalue problem that defines the next-order stresses and displacements to within a multiplicative constant, as well as the second $r$ exponent of the stress expansion. When there is substantial hardening, however, the elastic effects enter the solution to second order and the magnitude of the second term in the expansion of the solution is controlled by the $J$-integral.

Standard notation is used throughout. Boldface symbols denote tensors, the order of which is indicated by the context, and the summation convention is used for repeated Latin indices.

\section{Formulation of the Problem}

We consider the two-dimensional problem (plane strain or plane stress) of a crack in a homogeneous isotropic elastoplastic material.

The constitutive behavior of the continuum is described by the $J_{2}$-deformation theory for a Ramberg-Osgood uniaxial stress-strain behavior, namely

$$
s_{i j}=\frac{1+v}{E} s_{i j}+\frac{1-2 v}{3 E} \sigma_{k k} \delta_{i j}+\frac{3}{2} \alpha \varepsilon_{0}\left(\frac{\sigma_{c}}{\sigma_{0}}\right)^{n}{ }^{1} s_{i j} \sigma_{0},
$$

where $\boldsymbol{\varepsilon}$ is the infinitesimal strain tensor, $\boldsymbol{\sigma}$ is the Cauchy stress tensor and $\mathbf{s}$ its 